\title{
Parental Support and Insecure Attachment Development: The Cortisol Stress Response as a Moderator
}

\author{
M. Houbrechts ${ }^{\mathrm{a}^{*}}$, B. Cuyvers ${ }^{\mathrm{a}}$, L. Goossens ${ }^{\mathrm{b}}$, P. Bijttebier ${ }^{\mathrm{b}}$, A. S. Bröhl ${ }^{\mathrm{b}}$, F. \\ Calders $^{\mathrm{c}}$, V. Chubar ${ }^{\mathrm{d}}$, S. Claes ${ }^{\mathrm{d}}$, F. Geukens ${ }^{\mathrm{b}}$, K. Van Leeuwen ${ }^{\mathrm{c}}$, W. Van Den \\ Noortgate $^{\text {ef }}$ S. Weyn ${ }^{\mathrm{b}}$ and G. Bosmans ${ }^{\mathrm{a}}$
}

${ }^{a}$ Clinical Psychology Research Group, KU Leuven (University of Leuven), Leuven, Belgium, ${ }^{b}$ School Psychology and Development in Context Research Unit, KU Leuven (University of Leuven), Leuven, Belgium, ${ }^{c}$ Parenting and Special Education Research Unit, KU Leuven (University of Leuven), Leuven, Belgium, ${ }^{d}$ Mind-body Research Unit, KU Leuven (University of Leuven), Belgium, ${ }^{e}$ Methodology of Educational Sciences Research Group \& imec-ITEC, KU Leuven (University of Leuven), Kortrijk, Belgium.

${ }^{*}$ Corresponding author: Melisse Houbrechts, KU Leuven, Tiensestraat 102, 3000 Leuven, Belgium, melisse.houbrechts@kuleuven.be, 0032 (0)16 193994. 


\begin{abstract}
The current study investigated whether variations at the level of the cortisol stress response moderate the association between parental support and attachment development. To test this hypothesis, we conducted a one-year longitudinal study with two waves in which 101 children $\left(56 \%\right.$ girls, $\left.M_{\mathrm{age}}=11.15, S D_{\mathrm{age}}=0.70\right)$ participated. Attachment anxiety and avoidance were measured at baseline (Wave 1) and one year later (Wave 2). Parental support and children's cortisol stress response during the Trier Social Stress Test were measured at Wave 2. Children's cortisol stress response was found to moderate the association between parental support and relative change in anxious attachment. A strong cortisol stress response weakened the associated between parental support and relative change in anxious attachment. No moderation effects were found for relative change in avoidant attachment.
\end{abstract}

Keywords: attachment; longitudinal; early adolescence; cortisol stress response; parental support 


\section{Parental Support and Insecure Attachment Development: The Cortisol Stress Response as a Moderator}

According to Bowlby (1969), the quality of children's attachment to their parents is mainly shaped by parental responses to the child's distress and support seeking behaviour. More specifically, parents of securely attached children are expected to respond more consistently in a supportive way to their children's distress. This allows children to rely more easily on their parents for support in times of distress. Parents of insecurely attached children are assumed to respond inconsistently or in an unsupportive way to their children's distress. As a result, children are expected to become insecurely attached and less able to rely on their parents in times of distress. Instead, they display heightened negative affect in an attempt to maintain parental support (i.e., anxious attachment) or they tend to avoid potential sources of distress and they reduce their support seeking behaviour (i.e., avoidant attachment) (Cassidy \& Shaver, 2016).

In spite of this straightforward theory, meta-analyses suggest that parental support is only partially explains attachment development in infancy (Van Ijzendoorn, 1995; Verhage et al., 2016). In middle childhood and adolescence, evidence for the link between parental support and attachment is even more mixed. Koehn and Kerns' meta-analysis (2018) found a medium sized link between parental support and avoidant attachment, and no association between parental support and anxious attachment. These findings seem to suggest that support explains only a limited amount of variance in attachment later in development.

To help explain this discrepancy between theory and research findings, Bosmans, Bakermans-Kranenburg, Vervliet, Verhees, and Van Ijzendoorn (2020) formulated a Learning Theory of Attachment (LTA). They propose that individual differences in attachment develop through safety conditioning. Each interaction with parents after children are exposed to a stressor is considered as a learning event. Through repeated learning events in which parents consistently provide support that reduces distress, parents can become associated with the 
expectation that distress will be regulated. At the neurobiological level, endocrine changes in response to parental support during stress are expected to underlie safety conditioning. For instance, cortisol reductions in response to parental support during stress (e.g. Gunnar \& Hostinar, 2015) are supposed to translate into a sense of relief. If parents consistently provide support that results in this endocrinologically-driven sense of relief, parents become associated with relief in times of distress (Bosmans et al., 2020).

According to the LTA, attachment learning occurs already early in development resulting in individual differences in attachment that become increasingly stable over time (Bosmans et al., 2020). However, attachment learning and resulting changes in attachment are still expected to occur later in development. This prediction is in line with longitudinal research showing limited stability of attachment over time (Groh et al., 2014) and research suggesting that attachment develops in a prototype fashion (i.e. early attachment experiences have a stable influence on attachment across time and attachment can be updated based on new experiences) (e.g. Jones et al., 2018). Thus, theory and research suggest that it remains relevant to identify factors that are associated with changes in attachment that occur later in development. One relevant developmental phase to study change in attachment is early adolescence because longitudinal research shows that at this age substantial changes in attachment can be observed (e.g. Khan et al., 2019).

The LTA also proposes that variation at the level of the endocrine systems involved in attachment development affects the likelihood that parental support will be associated with change in attachment over time (Bosmans et al., 2020). The current study focusses on variability at the level of the cortisol stress response. Children with a stronger cortisol response are harder to regulate. As a result, they are expected to be less likely to experience that parental support successfully reduces distress. Consequently, these children are expected to learn less easily that parents can be a safety signal that predicts comfort during distress. 
The cortisol stress response is coordinated by the hypothalamic pituitary adrenal (HPA) axis (Gunnar \& Quevedo, 2007). Following exposure to distress, a hormonal cascade in the HPA-axis stimulates production of cortisol, which in turn prepares the body to cope physiologically and behaviourally with distress. The cortisol stress response typically consists of three successive components: (a) an increase in cortisol which is labelled cortisol reactivity and reflects the magnitude of the activation in the stress response system after exposure to a stressor, (b) a peak in cortisol levels, and (c) a decline in cortisol which is labelled cortisol recovery and reflects the rate of return to baseline cortisol. The cortisol stress response can vary from hyper- to hypoactivation. This variation is not only affected by inborn genetic factors, but also by environmental factors that occur early in development such as adversity, early attachment, or caregiving history (Gunnar, Doom, \& Esposito, 2015).

In the current study, we will test whether variations in the three components of the cortisol stress response moderate the link between parental support and insecure attachment development. We hypothesize that the association between parental support and decreases in insecure attachment is weaker when children display a stronger cortisol response to stress (as reflected by high levels of reactivity, high peak levels, and low levels of recovery) because this reduces the likelihood that children experience distress reductions following parental support. This hypothesis will be tested separately for relative change in anxious (Hypothesis 1) and avoidant attachment (Hypothesis 2). Based on Koehn and Kerns' (2018) finding that parental support was more strongly associated with avoidant attachment compared to anxious attachment, we expect to find more evidence for a moderation effect when predicting relative change in anxious attachment. Variation in the cortisol stress response could help explain why parental support is not for all children associated with relative change in anxious attachment. 


\section{Methods}

\section{Subjects}

The study sample is a subsample of the Methylation in Development (MIND) study, a longitudinal study on social and emotional development during early adolescence conducted in Belgium. Early adolescents and their parents were informed about the study through flyers distributed in schools, public spaces and on social media. At Wave 2, the participants were invited to participate in a more intensive laboratory assessment. The sample consisted of 101 children (45 boys, 56 girls) between 9 and 12 years old $(M=10.18 ; S D=0.70)$ at Wave 1 . Most children came from intact families $85.1 \%, 11.9 \%$ had divorced parents and $2.0 \%$ had a deceased biological father. Regarding nationality, $91.9 \%$ had the Belgian nationality and 6.9 $\%$ had a different (additional) nationality (1 Chinese, 1 Rumanian, 1 Ecuadorian, 2 Dutch, 1 Finnish, and 1 Russian). In the current sample, $84.5 \%$ of the adolescents had a mother with a bachelor's degree or higher.

\section{Procedure}

For both waves, parents completed an informed consent prior to data collection. At Wave 1, the children participated in their classroom or came to the research centre to fill out questionnaires that were part of the broader research project. At Wave 2, all participants came to the research centre to complete the same questionnaires and to participate in a modified version of the Trier Social Stress Test (TSST-M). Two saliva samples were collected at 50 minutes and 2 minutes before the start of the TSST-M. The first sample was collected after the consent procedure and the second sample was collected before the participants moved to the room where they participated in the TSST-M. The first sample was not included in the analyses because it was collected immediately after arrival to the lab solely to examine impact of circumstances prior to the lab visit (e.g. vigorous physical activity) on cortisol. However, 
children typically experience high levels of stress when entering the strange situation of a laboratory setting. Therefore, cortisol levels at this moment do not reflect children's baseline cortisol in the absence of stress. The sample collected two minutes before the TSST-M was used as baseline. After the TSST-M, they sat quietly in a room for 50 minutes and could play with toys. Six saliva samples were collected at 20, 30, 40, 50, 60, and 70 minutes after the start of the TSST-M. Two hours after the end of the TSST-M, participants filled out questionnaires measuring perceived parental support and insecure attachment appraisals. Parents reported on children's medication use and pubertal status. After completion, participants were fully debriefed and received a modest gift. The procedure was approved by the local university's ethical committee.

\section{Measures}

\section{Insecure Attachment}

Children's insecure attachment appraisals about the mother were assessed using the 12-item Experience in Close Relationships Scale - Revised Child Version (ECR-RC, Brenning, Soenens, Braet, \& Bal, 2012). It consists of six items that measure attachment anxiety (e.g., I'm worried that my mother might want to leave me) and six items that measure attachment avoidance (e.g., I prefer not to get too close to my mother). Children rated each item on a 7-point scale from 1 (= strongly disagree) to 7 (= strongly agree). Scores on items that measure anxious and avoidant attachment were summed to obtain an anxiety and avoidance score, respectively, with higher scores representing more anxious or avoidant attachment. Supporting the validity of the brief ECR-RC, both scale scores were associated with observed support seeking behaviour (Dujardin et al., 2016). In the present study, Cronbach's alphas indicated a sufficient internal consistency $\left(\alpha_{\text {anxiety } \text { wave } 1}=\right.$ $.90, \alpha_{\text {avoidance Wave } 1}=.69, \alpha_{\text {anxiety Wave } 2}=.87, \alpha_{\text {avoidance Wave } 2}=.73$ ). 


\section{Parental Support}

The Support subscale of the Parenting Scale was used to assess the extent to which children perceived their parents as involved, accepting, emotionally available, and responsive to their needs (Janssens et al., 2015). The Support subscale consists of 23 items (e.g., My parents make me feel better when I am feeling upset). Children simultaneously reported about both parents. Strong correlations between child reports of support provided by mothers and fathers justify this approach (Van Leeuwen, Mervielde, Braet, \& Bosmans, 2004). Children rated items on a 5-point scale ranging from 1 (=almost never) to 5 (=almost always). The Support subscale is a reliable and valid scale to assess perceived parental support (Spithoven, Bijttebier, \& Leeuwen, 2016). Item scores were summed, with higher scores representing higher levels of perceived parental support. The internal consistency at Wave 2 was good $(\alpha=.94)$

\section{TSST-M}

The TSST-M is a 15-minute, age-appropriate laboratory stressor task that reliably elicits a cortisol stress response in children (Yim, Quas, Cahill, \& Hayakawa, 2010). In the testing room, two female observers wearing lab coats were seated and a video camera was positioned in prominent view. The lead researcher explained to the participants that they had to deliver a speech for which they were given 3 minutes preparation time, and that they would perform a math task as well. They were also informed that videotapes were made that would be shown to experts and that the observers would analyse their behaviours. The speech task (6 minutes) consisted of a role play in which participants had to introduce themselves to a new classroom and was explained by one observer. During the speech task and math task, the observers were non-responsive. The math task (4 minutes), which consisted of a serial subtraction task (beginning by subtracting 5 from 1027), was explained by the other observer. The observer instructed the participants that they had to perform the math task as fast and 
accurate as possible. Every time they made a mistake, they were corrected by the observer and were asked to start over.

\section{Salivary Collection and Analysis}

Children were asked to abstain from food, drinks, and vigorous physical exercise two hours prior to the testing sessions. Appointments were scheduled in the early afternoon (between 2:00 and 3:00 p.m.) to keep circadian variation in cortisol levels constant. Saliva samples were collected using salivettes (Salivettes, Sarstedt), stored at room temperature until completion of the experiment and then kept at $-20^{\circ} \mathrm{C}$. After thawing, the salivettes were centrifuged at 3,000 rpm for 5 minutes. Next, cortisol concentrations were measured using commercially available chemiluminescence immunoassay with high sensitivity (IBL International, Hamburg, Germany). All samples were analysed in duplicates. The intra- and inter-assay coefficients for cortisol were below 7\%. The average of duplicate assays was used for further analysis.

\section{Covariates}

In our analyses, we included children's gender (boy =1, girl =2), age, pubertal status, and medication use as covariates because these variables have been linked before to individual differences in the cortisol stress response (Granger, Hibel, Fortunato, \& Kapelewski, 2009). Pubertal status was measured using the Dutch version of the Pubertal Development Scale (Petersen, Crockett, Richards, \& Boxer, 1988), with higher scores reflecting more advanced pubertal status. Parent-reported medication use was coded 1 if it could influence cortisol collection and assessment and 0 if it could not. We also controlled for the other insecure attachment style and the components of the cortisol stress response to evaluate the extent to which effects were unique for each insecure attachment style and cortisol stress response component. 


\section{Data Analytic Plan}

Raw cortisol values were log transformed because the distribution was positively skewed. In the current study, we used a bilinear spline model with an individually varying peak time to extract estimates of the cortisol peak values, reactivity, and recovery for each participant (a detailed description is presented in Supplementary Material 1). This decision was based on the observation that the current sample varied substantially in the timing of the peak. This analysis allowed estimating the components of the cortisol stress response separately. This enabled a fine-grained analyses of variations in the cortisol stress response and prevented loss of information that occurs when a single index is used to quantify the cortisol stress response such as the Area under the Curve (AUC). Nevertheless, to allow comparison with other studies, we described the AUC analyses in Supplementary Material 2. The AUC analyses showed similar interactions effects.

To test our hypotheses that variations in the components of the cortisol stress response moderate the effect of parental support on relative change in anxious attachment, we performed multiple hierarchical regression analyses. In Step 1, Wave 2 anxious attachment was regressed on support and cortisol reactivity/ peak/ recovery while controlling for Wave 1 anxious attachment (to predict relative change one year after baseline). The Support x Cortisol interaction was added in Step 2. Interactions between parental support and each component of the cortisol stress response were tested in separate analyses. Significant interaction effects were followed up by simple slope analyses testing the slope of support at low and high cortisol levels (1 $S D$ below and above the mean). Regions of significance analyses using the Johnson-Neyman technique were also performed to identify the levels of the moderator at which support was significantly associated with increases in insecure attachment. All analyses were repeated controlling for the covariates and with pair-wise deletion to more 
conservatively handle missing values. Similar analyses were performed to predict relative change in avoidant attachment.

\section{Results}

\section{Missing Values}

Little's MCAR test did not reveal significant evidence against the hypothesis that the missingness $(5.6 \%)$ was completely random $\left(\chi^{2}(119)=135.090, p=.149\right)$. Expectation Maximization was used to handle missing data. Descriptives are presented in Supplementary Material 3.

[Table 1 near here]

\section{Cortisol X Parental Support and Change in Anxious Attachment}

For the analysis with reactivity as a moderator, there was a significant interaction (Table 2, Figure 1a). Simple slope analysis showed that more parental support was significantly associated with decreases in anxious attachment, but this slope was more negative for children with lower levels of reactivity. The difference between children with high versus low reactivity was significant when support was low but not when support was high. When support was low, children with lower reactivity showed larger increases in anxious attachment compared to children with higher reactivity. The regions of significance analysis indicated that higher levels of parental support predicted decreases in anxious attachment when reactivity was below 1.14 (z-score).

Results of the analysis with cortisol peak as moderator showed a similar interaction effect, fostering the same straightforward interpretation. In view of space constrains in this special issue, the results are presented in Supplementary Material 4. For the analysis with recovery as a moderator, there was a significant interaction (Table 2, Figure 1b). Simple slope analysis showed that more parental support was associated with decreases in anxious 
attachment, but the slope was more negative for children with low levels of recovery compared to children with high levels of recovery. The difference between children with high versus low recovery was significant when support was high but not when support was low. Children with lower recovery showed larger decreases in anxious attachment compared to children with higher recovery. The regions of significance analysis indicated that higher levels of parental support predicted decreases in anxious attachment when recovery was above -1.28 (z-score).

[Table $2 \&$ Figure 1 near here]

After controlling for the covariates, the three interaction effects remained significant (Reactivity x Support: $\beta=.285, p=.001$; Peak x Support: $\beta=.287, p<.001$; Recovery $\mathrm{x}$ Support: $\beta=-.243, p=.004)$. In all the simple slope analyses, the slopes of support were no longer statistically significant for children with high cortisol reactivity, peak and recovery levels $\left(B_{\text {high reactivity }}=-0.046, t(90)=-0.892, p=.375 ; B_{\text {high peak }}=-0.052, t(90)=-1.030\right.$, $\left.p=.301 ; B_{\text {high recovery }}=-0.071, t(90)=-1.379, p=.171\right)$. In the analysis using pairwise deletion to handle missing data, the three interaction effects remained significant (Reactivity $\mathrm{x}$ Support: $\beta=.326, p=.001$; Peak x Support: $\beta=.506, p=.003$; Recovery x Support: $\beta=$ $.240, p=.010)$. The slopes also no longer reached significance at high cortisol reactivity, peak and recovery levels. We also conducted a follow-up analysis which simultaneously included all three interaction effects. Results of this analysis showed that none of the interaction effects remained significant (Support $\mathrm{x}$ Reactivity: $\beta=.157, \mathrm{p}=.177$; Support $\mathrm{x}$ Peak levels: $\beta=$ $.172, p=.216 ;$ Support $x$ Recovery: $\beta=-.037, p=.781)^{1}$.

\section{Cortisol X Parental Support and Change in Avoidant Attachment}

\footnotetext{
${ }^{1}$ Additional information about these analyses can be requested from the corresponding author.
} 
All three interaction effects were non-significant (Reactivity x Support: $\beta=-.021$, $t(96)=-0.228, p=.820$; Peak levels $x$ Support: $\beta=-.003, t(96)=-0.031, p=.975$; Recovery x Support: $\beta=-.064, t(96)=-0.746, p=.458)$. Adding covariates and repeating the analyses treating missing data with pairwise deletion did not alter these conclusions ${ }^{2}$.

\section{Discussion}

The current study aimed to investigate whether variations in the cortisol stress response moderate the association between parental support and relative change in insecure attachment. Based on the LTA, we hypothesized that the association between parental support and relative change in insecure attachment would be weaker when children display a stronger cortisol stress response. The results supported our hypotheses when predicting relative change in anxious attachment, but not when predicting relative change in avoidant attachment.

\section{Cortisol X Parental Support and Change in Anxious Attachment}

In line with our predictions, a strong cortisol stress response (high reactivity, peak levels and recovery) weakened the association between support and relative change in anxious attachment. The results were replicated over all three components of the cortisol stress response. This makes sense as a strong cortisol response typically means high reactivity, high peak levels, and high recovery and the associations reported in Table 1 indeed suggest that the components are strongly correlated. The effects also survived controlling for the covariates and were not dependent on statistical analysis strategies. This further supported the robustness of the findings. As predicted, these findings suggest that a strong cortisol stress response reduces the overall effect of support on anxious attachment development during early adolescence.

\footnotetext{
${ }^{2}$ Additional information about these analyses can be requested from the corresponding author.
} 
We cannot firmly conclude that the effect we found explains early adolescents' attachment development because relative change in attachment in the current study might merely reflect error variance. The increasingly supported prototype model of attachment (in)stability suggests that early attachment representations continue to influence attachment later in life and that attachment becomes an increasingly stable feature (Fraley, 2002). However, according to the prototype model attachment can still be updated based on novel experiences. Research indeed shows that significant changes in attachment occur during early adolescence (Groh et al., 2014) and that these changes are associated with meaningful environmental factors (Waters et al., 2019). In line with this research, our findings suggest that changes in attachment during early adolescence are meaningfully associated with parenting. The current study also adds to this literature that a strong cortisol stress response decreases the impact of parenting on changes in attachment.

At this point, we cannot infer from the data that we demonstrated an ongoing interaction between parenting, the cortisol stress response, and attachment in early adolescence. These factors might have already reciprocally affected each other's development in early childhood (or even prenatally), for instance through methylation of the glucocorticoid receptor genes involved in the regulation of the cortisol stress response (Turecki \& Meaney, 2016). In the light of this reasoning, it is important to note that Bosmans, Young, and Hankin (2018) also found that methylation in the glucocorticoid receptor gene moderated the effect of parental support during distress on anxious attachment development during early adolescence. So, it is important to test whether this effect survives controlling for early attachment and stress exposure before we can conclude that the interaction effects explain variation in early adolescents' attachment development. Nevertheless, it is promising that these findings support our hypothesis that children with a strong cortisol stress response are less likely to learn from (a lack of) parental support. In line with our predictions, it could indeed be that for these 
children parental support has a less reinforcing effect in terms of stress reduction and that, therefore, parents are less likely to become associated with (a lack of) comfort during distress. Interestingly, children with high levels of reactivity and peak showed smaller increases in anxious attachment when support was low compared to children with lower reactivity and peak levels. One interpretation could be that an enhanced cortisol stress response protects children against the negative effect of low parental support on their attachment development. Such a protective effect is in line with research suggesting that a strong cortisol response might have an adaptive value for children exposed to a stressful and unsupportive environment (Del Giudice, Ellis, \& Shirtcliff, 2011). This is also in line with theories suggesting that the impact of parenting on development depends on the extent to which it (mis)matches with children's phenotype that is shaped by their early environment (Nederhof \& Schmidt, 2012). Arguing against this protective effect interpretation, a strong cortisol response could not fully erase increases in anxious attachment associated with low support. Moreover, the effect was not robust as it was not replicated for cortisol recovery. For now, the safest interpretation is that, attachment development of children with a strong cortisol stress response is also less influenced by low parental support.

\section{Cortisol X Parental Support and Change in Avoidant Attachment}

In contrast to our second hypothesis, none of the cortisol stress response components moderated the link between parental support and relative change in avoidant attachment. In line with these findings, Bosmans et al. (2018) also failed to find moderating effects of (epigenetically-related) cortisol stress response system variation on the link between parental support and changes in avoidant attachment in a comparable age-group. However, the fact that there were no significant interaction effects could also be attributed to use of self-report measures for assessing avoidant attachment. Research suggests that avoidantly attached adolescents underreport levels of avoidant attachment relative to their mental representations 
(Borelli et al., 2016; Venta, Shmueli-Goetz, \& Sharp, 2014) and that they are inclined to dismiss negative (emotional) information (Cassidy \& Shaver, 2016). It is possible that in our sample attachment avoidance was underreported or that dismissing response styles affected the validity of some children's reports of parental support. This might indicate that the use of self-report questionnaires is a drawback of the current study. If these findings can be replicated using other measures than self-report measures to assess insecure attachment, it might be worthwhile to think of alternative explanations. For example, other neuroendocrine systems might play a role in avoidant attachment development such as the dopamine, oxytocin, and opioid systems (Vrtička, 2017). Replicating the current study including other hormones could be a promising avenue for future research.

\section{Limitations and Concluding Remarks}

Findings of the current study need to be considered in the context of several limitations. Insecure attachment was measured using self-report questionnaires that only tap into a more deliberate component of the attachment system (Bosmans \& Kerns, 2015). It is important that future work uses attachment measures that asses more automatic processes of the attachment system such as the Child Attachment Interview (Borelli et al., 2016). Nevertheless, significant associations between self-reported insecure attachment and psychosocial adjustment (Madigan, Brumariu, Villani, Atkinson, \& Lyons-Ruth, 2016) support the relevance of self-reported insecure attachment styles as predictor of the development of psychopathology. Furthermore, we solely relied on adolescent reports for parental support and insecure attachment. Our results might therefore be inflated by monoinformant bias. Future studies should replicate these results using multiple-informant reports (i.e., parent and adolescent reports) and/or observational measures to further strengthen these findings. Another limitation is that we only focused on insecure attachment to the mother, we did not measure insecure attachment appraisals about the father. Future studies need to test 
whether the cortisol stress response affects the development of attachment to the father in a similar way. Additionally, our predictor variables were assessed at Wave 2 and not at Wave 1 because prior research and theory suggests that it is relevant to use ongoing parenting as predictor of ongoing change in attachment (Bosmans et al., 2020; Finet et al., 2020). Also, the question can be raised whether the changes in insecure attachment reflected attachment development. Instead, these changes might have been induced by our manipulation as the Wave 2 questionnaires were administered after the TSST-M. The resulting procedural differences between both waves could have resulted in different response styles at each wave. The stress induction might also have influenced children's reports about parental support. However, it should be noted that cortisol levels typically return to baseline 60 minutes after the stressor (Labuschagne, Grace, Rendell, Terrett, \& Heinrichs, 2019). In the current study, children completed the questionnaires two hours after the end of the TSST-M, which reduced the likelihood that the children were still stressed when they completed the questionnaires. Furthermore, increases in cortisol relative to baseline and the extent to which cortisol levels returned to baseline levels were not associated with changes in attachment. This also suggests that it is unlikely that stress can account for the changes in attachment. Nevertheless, future studies might consider administering the TSST-M and the questionnaires on separate measurement occasions to further control for effects of design on the results. Finally, our sample was relatively small and consisted of children from a European country with a high socio-economic status. It is unclear, therefore, to which degree our findings can be generalized to early adolescents from other countries or with a less privileged background. In spite of these limitations, the current findings add to the literature on attachment development. Whereas this literature is traditionally strongly focused on parenting to explain attachment development, we showed that factors at the biological level might moderate the association between parental support and relative change in anxious attachment. Our results 
supported a core prediction of the recently formulated LTA, suggesting that this might be a promising avenue for a more coherent understanding of individual differences in attachment development.

\section{Declaration of interest}

None.

\section{Acknowledgement}

This work was supported by the $[\mathrm{XXXXX}]$ under Grant $[\mathrm{XXXXX}]$ and $[\mathrm{XXXXX}]$ under Grant $[\mathrm{XXXXX]}$.

\section{References}

Borelli, J. L., Somers, J., West, J. L., Coffey, J. K., De Los Reyes, A., \& Shmueli-Goetz, Y. (2016). Associations Between Attachment Narratives and Self-Report Measures of Attachment in Middle Childhood: Extending Evidence for the Validity of the Child Attachment Interview. Journal of Child and Family Studies, 25, 1235-1246. https://doi.org/10.1007/s10826-015-0310-8

Bosmans, G., Bakermans-Kranenburg, M. J., Vervliet, B., Verhees, M. W. F. T., \& Van Ijzendoorn, M. H. (2020). A learning theory of attachment: Unraveling the black box of attachment development. Neuroscience \& Biobehavioral Reviews, 113, 287-298. https://doi.org/10.1016/j.neubiorev.2020.03.014

Bosmans, G., \& Kerns, K. A. (2015). Attachment in middle childhood: progress and prospects. In G. Bosmans \& K. A. Kerns (Eds.), Attachment in middle childhood: Theoretical advances and new directions in an emerging field. (Vol. 148, pp. 1-14). https://doi.org/10.1002/cad

Bosmans, G., Young, J., \& Hankin, B. (2018). NR3C1 methylation as a moderator of the effects of maternal support and stress on insecure attachment development. Developmental Psychology, 54, 29-38. https://doi.org/10.1037/dev0000422.NR3C1 Bowlby, J. (1969). Attachment and loss. (Vol. I, Attachment). New York: NY: Basic Books. Brenning, K., Soenens, B., Braet, C., \& Bal, S. (2012). The Role of Parenting and MotherAdolescent Attachment in the Intergenerational Similarity of Internalizing Symptoms. Journal of Youth and Adolescence, 41, 802-816. https://doi.org/10.1007/s10964-011- 
9740-9

Cassidy, J., \& Shaver, P. R. (Eds.). (2016). Handbook of attachment: Research, theory and clinical applications. (Third). New York: Guilford Press.

Del Giudice, M., Ellis, B. J., \& Shirtcliff, E. A. (2011). The Adaptive Calibration Model of stress responsivity. Neuroscience and Biobehavioral Reviews, 35, 1562-1592. https://doi.org/10.1016/j.neubiorev.2010.11.007

Dujardin, A., Santens, T., Braet, C., De Raedt, RudiVos, P., Maes, B., \& Bosmans, G. (2016). Middle Childhood Support-Seeking Behavior During Stress : Links With Self-Reported Attachment and Future Depressive Symptoms. Child Development, 87, 326-340. https://doi.org/10.1111/cdev.12491

Finet, C., Waters, T. E. A., Vermeer, H. J., Juffer, F., Van IJzendoorn, M. H., BakermansKranenburg, M. J., \& Bosmans, G. (2020). Attachment development in children adopted from China: the role of pre-adoption care and sensitive adoptive parenting. Attachment and Human Development, 1-21. https://doi.org/10.1080/14616734.2020.1760902

Fraley, R. C. (2002). Attachment Stability from Infancy to Adulthood: Meta-Analysis and Dynamic Modeling of Developmental Mechanisms. Personality and Social Psychology Review, 6, 123-151. https://doi.org/10.1207/s15327957pspr0602_03

Granger, D. A., Hibel, L. C., Fortunato, C. K., \& Kapelewski, C. H. (2009). Medication effects on salivary cortisol: Tactics and strategy to minimize impact in behavioral and developmental science. Psychoneuroendocrinology, 34, 1437-1448. https://doi.org/10.1016/J.PSYNEUEN.2009.06.017

Groh, A. M., Roisman, G. I., Booth-Laforce, C., Fraley, R. C., Owen, M. T., Cox, M. J., \& Burchinal, M. R. (2014). IV. Stability of attachment security from infancy to late adolescence. Monographs of the Society for Research in Child Development, 79, 51-66. https://doi.org/10.1111/mono.12113

Gunnar, M. R., Doom, J. R., \& Esposito, E. A. (2015). Psychoneuroendocrinology of Stress: Normaitve Development and Individual Differences. In M. E. Lamb \& R. M. Lerner (Eds.), Handbook of Child Psychology and Developmental Science: Socioemotional processes (7th ed., pp. 106-143). https://doi.org/10.1002/9781118963418.childpsy304

Gunnar, M. R., \& Hostinar, C. E. (2015). The social buffering of the hypothalamic-pituitaryadrenocortical axis in humans: Developmental and experiential determinants. Social Neuroscience, 10, 479-488. https://doi.org/10.1080/17470919.2015.1070747

Gunnar, M. R., \& Quevedo, K. (2007). The Neurobiology of Stress and Development. Annual 
Review of Psychology, 58, 145-173.

https://doi.org/10.1146/annurev.psych.58.110405.085605

Jones, J. D., Fraley, R. C., Ehrlich, K. B., Stern, J. A., Lejuez, C. W., Shaver, P. R., \& Cassidy, J. (2018). Stability of Attachment Style in Adolescence: An Empirical Test of Alternative Developmental Processes. Child Development, 89, 871-880. https://doi.org/10.1111/cdev.12775

Khan, F., Chong, J. Y., Theisen, J. C., Fraley, R. C., Young, J. F., \& Hankin, B. L. (2019). Development and Change in Attachment: A Multiwave Assessment of Attachment and Its Correlates Across Childhood and Adolescence. Journal of Personality and Social Psychology. https://doi.org/10.1037/pspi0000211

Koehn, A. J., \& Kerns, K. A. (2018). Parent-child attachment: meta-analysis of associations with parenting behaviors in middle childhood and adolescence. Attachment and Human Development, 20(4), 378-405. https://doi.org/10.1080/14616734.2017.1408131

Labuschagne, I., Grace, C., Rendell, P., Terrett, G., \& Heinrichs, M. (2019). An introductory guide to conducting the Trier Social Stress Test. Neuroscience and Biobehavioral Reviews, 107(September), 686-695. https://doi.org/10.1016/j.neubiorev.2019.09.032

Madigan, S., Brumariu, L. E., Villani, V., Atkinson, L., \& Lyons-Ruth, K. (2016). Representational and questionnaire measures of attachment: A meta-analysis of relations to child internalizing and externalizing problems. Psychological Bulletin, 142, 367-399. https://doi.org/10.1037/bul0000029

Nederhof, E., \& Schmidt, M. V. (2012). Mismatch or cumulative stress: Toward an integrated hypothesis of programming effects. Physiology and Behavior, 106, 691-700. https://doi.org/10.1016/j.physbeh.2011.12.008

Petersen, A. C., Crockett, L., Richards, M., \& Boxer, A. (1988). A self-report measure of pubertal status: Reliability, validity, and initial norms. Journal of Youth and Adolescence, 17, 117-133. https://doi.org/10.1007/BF01537962

Spithoven, A. W. M., Bijttebier, P., \& Leeuwen, K. Van. (2016). Brief report : Factor structure of parenting behaviour in early adolescence. Journal of Adolescence, 53, 9194. https://doi.org/10.1016/j.adolescence.2016.09.002

Turecki, G., \& Meaney, M. J. (2016). Effects of the Social Environment and Stress on Glucocorticoid Receptor Gene Methylation: A Systematic Review. Society of Biological Psychiatry, 79, 87-96. https://doi.org/10.1016/j.biopsych.2014.11.022

Van Ijzendoorn, M. H. (1995). Adult attachment representations, parental responsiveness, and 
infant attachment: A meta-analysis on the predictive validity of the adult attachment interview. Psychological Bulletin, 117, 387-403. https://doi.org/10.1037/00332909.117.3.387

Van Leeuwen, K., Mervielde, I., Braet, C., \& Bosmans, G. (2004). Child personality and parental behavior as moderators of problem behavior: Variable- and person-centered approaches. Developmental Psychology, 40, 1028-1046. https://doi.org/10.1037/00121649.40.6.1028

Verhage, M. L., Schuengel, C., Madigan, S., Fearon, P. R. M., Oosterman, M., Cassibba, R., ... van IJzerdoorn, M. H. (2016). Narrowing the transmission gap: a synthesis of three decades of research on intergenerational transmission of attachment. Psychological Bulletin, 142, 337-366. https://doi.org/10.1037/bul0000038

Vrtička, P. (2017). The social neuroscience of attachment. In A. Ibáñez, L. Sedeño, \& A. M. García (Eds.), Neuroscience and social science (pp. 95-119). https://doi.org/10.1007/978-3-319-68421-5_5

Waters, T. E. A., Facompré, C. R., Van de Walle, M., Dujardin, A., De Winter, S., Heylen, J., ... Bosmans, G. (2019). Stability and Change in Secure Base Script Knowledge During Middle Childhood and Early Adolescence: A 3- Year Longitudinal Study. Developmental Psychology. https://doi.org/10.1037/dev0000798

Yim, I. S., Quas, J. A., Cahill, L., \& Hayakawa, C. M. (2010). Children's and adults' salivary cortisol responses to an identical psychosocial laboratory stressor. Psychoneuroendocrinology, 35, 241-248. https://doi.org/10.1016/J.PSYNEUEN.2009.06.014 
Table 2. Multiple regression analyses testing the interactions between cortisol reactivity/ recovery and parental support to predict relative change in anxious attachment.

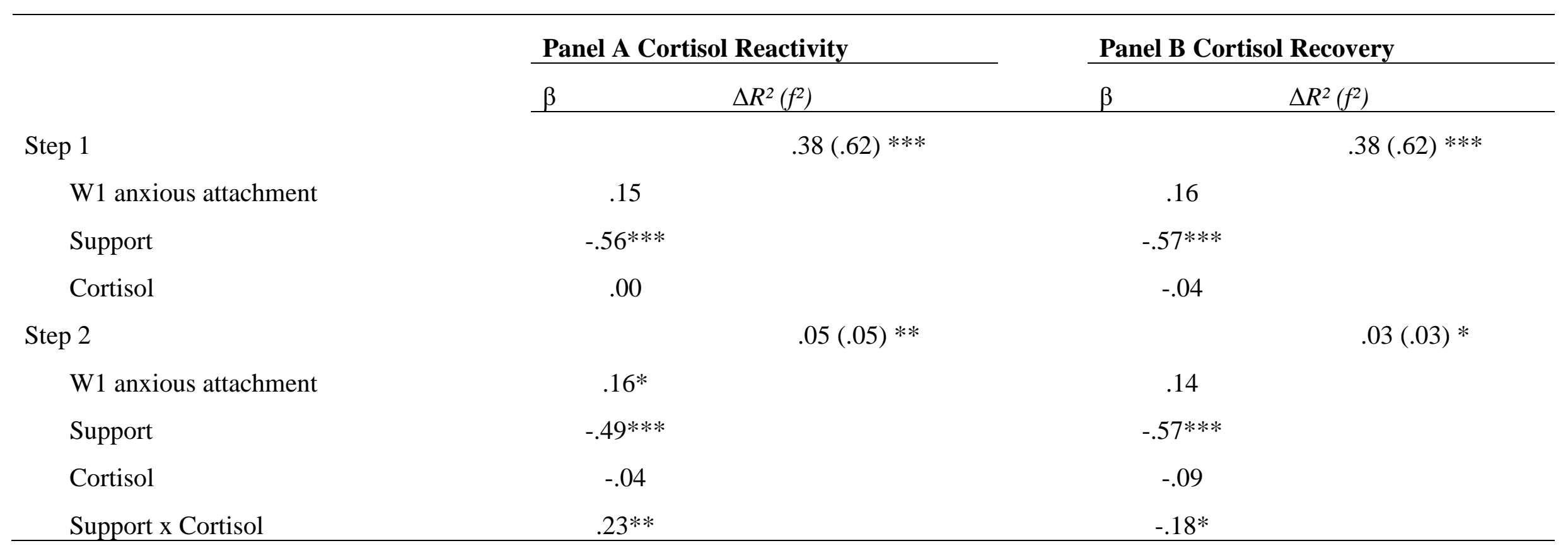

$* p<.05, * * p<.01, * * * p<.001$

Note: $\mathrm{W} 1=$ Wave 1 
Panel a: Reactivity X Support

0

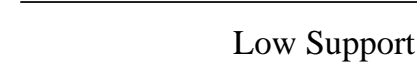

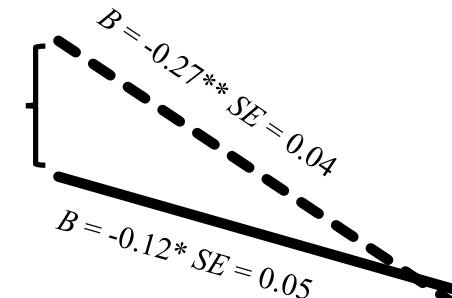

$\rightarrow$

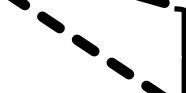

$B=76.63$

$S E=46.60$

$\boldsymbol{- \bullet} \bullet$ Low reactivity High reactivity

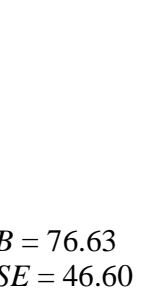

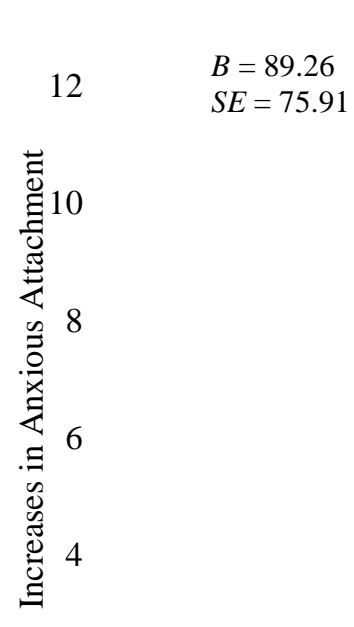

2
Panel b: Recovery X Support

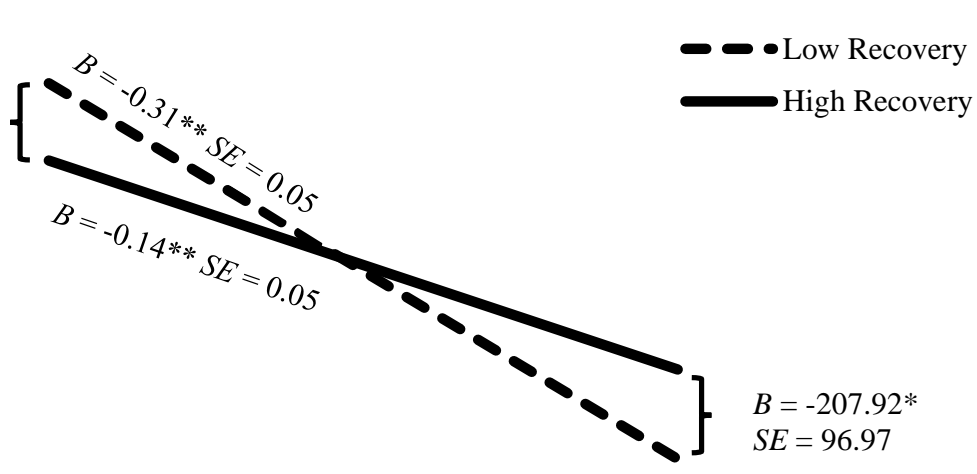

Figure 1. Interaction effects between parental support and cortisol reactivity/ recovery (Panel a: Cortisol Reactivity; Panel b: Cortisol Recovery) on relative change in attachment anxiety. 
